\title{
Le misure di associazione in medicina ed epidemiologia
}

\author{
Francesco Franco ${ }^{1}$, Anteo Di Napoli ${ }^{2}$ \\ ${ }^{1}$ Regione Lazio, Roma \\ ${ }^{2}$ Comitato Tecnico-Scientifico RIDT, Roma
}

\begin{abstract}
Measures of association in medicine and epidemiology
The measures of association evaluate the direction and magnitude of the relationship between two variables. The "effect size" of the association is described with a variety of coefficients when variables are expressed as continuous data (e.g. Pearson correlation coefficient), while if the association refers to categorical variables, the strength of the relationship between these variables is measured through difference in proportions, relative risk, odds ratio. Tests of significance evaluate statistically whether the relationship between the variables is significant or not.
\end{abstract}

Keywords: Association, Correlation, Number needed to treat, Odds ratio, Relative risk, Risk difference

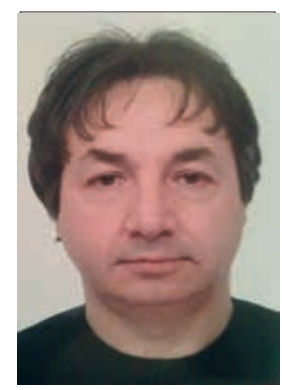

Franco Francesco
Dopo il ciclo di articoli dedicati alla validità e alla riproducibilità delle misure (1-5), diamo inizio a una nuova serie, relativa alle misure di associazione, con particolare riferimento a quelle più utilizzate in campo medico ed epidemiologico. Nel presente manoscritto ci limiteremo a passare in rassegna le principali grandezze statistiche della misura del rischio che saranno riprese e approfondite nei successivi articoli, non prima di aver introdotto il concetto di associazione. Il termine associazione è ampiamente usato in letteratura scientifica e, in generale, esprime la relazione statistica tra due variabili in esame. Lo studio dell'associazione richiede almeno due livelli di analisi: il primo volto a valutare se la relazione possa essere spiegata dalla presenza di una terza variabile e il secondo volto a misurare l'associazione attraverso la definizione e la scelta della misura più adatta e a testare la sua significatività statistica (6). L'approccio epidemiologico consiste, prima di tutto, nello stabilire se esiste un'associazione statistica tra un'esposizione (caratteristica) e un esito (per esempio, una patologia) e, conseguentemente, nel verificare la veridicità dell'associazione (causale o eziologica) oppure

Accepted: April 1, 2017

Published online: April 21, 2017

Indirizzo per la corrispondenza: franco_francesco@fastwebnet.it anteo.dinapoli@libero.it nell'individuare un'associazione spuria (falsa, non causale). In linea di massima, l'obiettivo degli studi epidemiologici consiste nell'individuare e nello stimare l'effetto di un'esposizione su un rischio per la salute. Tuttavia, dal momento che non è quasi mai possibile misurare direttamente l'effetto, si misura, in sua vece, l'associazione tra l'esposizione e il rischio. Supponiamo di misurare l'occorrenza di una patologia in due diverse popolazioni, per esempio il tasso di incidenza di cancro tra maschi e femmine. Questo tasso di incidenza non è una misura diretta di effetto, ma una misura di associazione, nel caso specifico quella tra il genere e il cancro (7).

Nelle indagini scientifiche, la ricerca di relazioni causali fra fattori di rischio e patologie rappresenta spesso l'obiettivo principale di uno studio (8). Uno dei passi cruciali da compiere nell'investigazione del nesso causale fra un fattore di esposizione e una data malattia è quello, quindi, della misurazione della loro dipendenza statistica o associazione. Si parla di presenza di associazione fra due eventi quando la probabilità di occorrenza dell'uno varia con la frequenza di occorrenza dell'altro (covariazione).

La scoperta della presenza di un'associazione statistica significativa fra un evento e un fattore di esposizione non rappresenta però l'evidenza di una relazione causale (9); I'esistenza di un'associazione statistica significativa è, quindi, una condizione necessaria ma non sufficiente per stabilire l'esistenza di un nesso causale. Tuttavia, la forza dell'associazione rimane uno dei principali elementi a favore dell'esistenza di un potenziale nesso causale nella relazione fra fattore di rischio e malattia, come riportato nei classici nove criteri di causalità definiti da Bradford Hill. Per contro, la rilevazione di una debole relazione potrebbe essere solo opera del caso o, più probabilmente, l'effetto di fattori confonden- 
TABELLA I - Misure di effetto utilizzate in ambito medico ed epidemiologico

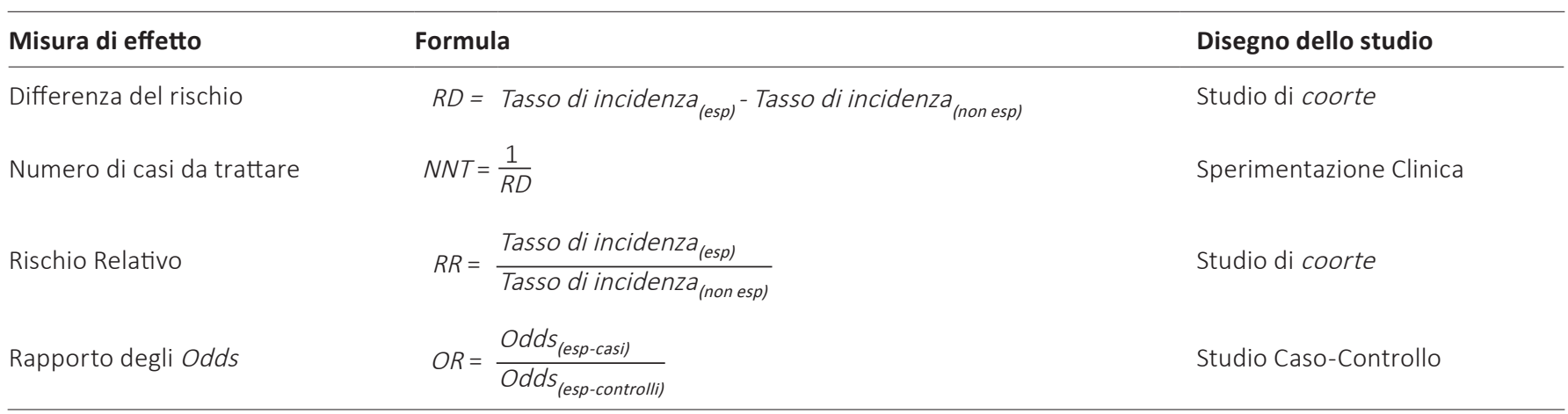

tiw la relazione tra esposizione e patologia (10).

La relazione di dipendenza (associazione) fra due variabili continue prende il nome di correlazione e uno degli indici che ne quantifica la forza è il coefficiente di correlazione lineare di Pearson; in caso di violazione dell'assunzione di normalità della distribuzione dei valori delle variabili in esame, il coefficiente di correlazione lineare di Pearson non è più applicabile. In tali circostanze, il coefficiente di correlazione per ranghi di Spearman è la misura non parametrica idonea per la quantificazione della forza della relazione.

Il test statistico del chi-quadrato è utilizzato, invece, per saggiare l'indipendenza di due variabili categoriche la cui distribuzione congiunta è riportata in una tabella di contingenza (11).

In ambito medico ed epidemiologico la presenza di associazione fra un fattore di esposizione e una malattia viene valutata analizzando la differenza di occorrenza della malattia nel gruppo dei soggetti esposti e in quello dei soggetti non esposti.

Quando si ha a che fare con misure di occorrenza si è soliti distinguere le misure di associazione in: misure basate sulla differenza assoluta del rischio e misure basate sulla differenza relativa del rischio. Le misure basate sulla differenza assoluta del rischio, quali la riduzione assoluta del rischio (ARR: Absolute Risk Reduction) conosciuta anche come differenza assoluta del rischio (RD: Risk Difference) e il numero dei casi da trattare per ottenere un esito positivo (NNT: Number Needed to Treat), sono utilizzate in sanità pubblica per quantificare l'impatto di un intervento (riduzione dei casi di malattia per effetto dell'eliminazione della causa).

Le misure basate sulla differenza relativa del rischio, quali il rischio relativo (RR: Relative Risk) e il rapporto fra gli odds (OR: Odds Ratio), forniscono, invece, la misura della forza di associazione fra fattore di esposizione e malattia. Gli epidemiologi utilizzano queste misure della dimensione di un effetto, "effect size", per la valutazione di potenziali associazioni causali (12). Queste misure non consentono la quantificazione dell'effetto di un intervento, in quanto questo dipende non solo dalla forza dell'associazione ma anche dalla prevalenza della patologia nella popolazione in studio.
Nella Tabella I sono riportate alcune misure di associazione utilizzate in ambito medico ed epidemiologico secondo il tipo di misura e il disegno dello studio.

\section{Disclosures}

Financial support: No financial support was received for this submission. Conflict of interest: The authors have no conflict of interest.

\section{Bibliografia}

1. Franco F, Di Napoli A. Introduzione alla valutazione di un test diagnostico: sensibilità, specificità, valore predittivo. Giornale di Tecniche Nefrologiche e Dialitiche. 2016;28(1):53-55.

2. Franco F, Di Napoli A. Rapporto di verosimiglianza del risultato positivo e negativo di un test diagnostico e teorema di Bayes. Giornale di Tecniche Nefrologiche e Dialitiche. 2016;28(2):134-136.

3. Franco F, Di Napoli A. Valutazione in parallelo e in serie di test diagnostici multipli. Giornale di Tecniche Nefrologiche e Dialitiche. 2016;28(3):212-214.

4. Franco F, Di Napoli A. Valutazione della riproducibilità di una misura: la statistica Kappa. Giornale di Tecniche Nefrologiche e Dialitiche. 2016;28(4):289-292.

5. Franco F, Di Napoli A. Valutazione della concordanza tra misurazioni di caratteri di tipo quantitativo: il metodo di BlandAltman. Giornale di Tecniche Nefrologiche e Dialitiche. 2017;29(1):59-61.

6. Woolson RF, Clarke WR. Statistical methods for the analysis of biomedical data, $2^{\text {nd }}$ edition. New York: Wiley \& Sons. 2002:263-266.

7. Rothman KJ, Greenland S. Modern epidemiology, $2^{\text {nd }}$ edition. Philadelphia: Lippincott Williams \& Wilkins. 1998:47-64.

8. Jekel JF, Katz DL, Elmore JG. Epidemiologia, biostatistica e medicina preventiva. Napoli: EdiSES. 2009:76-77.

9. Porta M. A Dictionary of Epidemiology, $6^{\text {th }}$ ed. New York: Oxford University Press. 2014:10.

10. Woodward M. Epidemiology. Study Design and Data Analysis, $2^{\text {nd }}$ edition. London: Chapman and Hall/CRC. 2005:19.

11. Everitt BS. Dizionario Cambridge di Statistica Medica. Roma: II Pensiero Scientifico Editore. 1998:287.

12. Szklo M, Nieto FJ. Epidemiology: beyond the basics. Gaithersburg, MD: Aspen Publishers. 2000:91-92. 